\title{
THE DYNAMICS OF THE GLOBE FOUNTAIN
}

\author{
S.J.D. D'ALESSIO ${ }^{1} \&$ J.P. PASCAL ${ }^{2}$ \\ ${ }^{1}$ Faculty of Mathematics, University of Waterloo, Canada \\ ${ }^{2}$ Department of Mathematics, Ryerson University, Canada
}

\begin{abstract}
We present results on the isothermal laminar flow of a thin fluid layer over a sphere as it exits from a small hole at the top of the sphere. Such a flow can be observed in a globe fountain. The fluid is taken to be viscous, incompressible and Newtonian while the flow is assumed to possess azimuthal symmetry. The governing Navier-Stokes equations are solved subject to no-slip and impermeability boundary conditions on the surface and the dynamic and kinematic conditions along the free surface. An approximate analytical solution for the steady-state flow has been derived by expanding the flow variables in powers of a small parameter, $\delta$, which represents the shallowness parameter. The leading and first-order terms in the series have been determined and the findings demonstrate that for thin flows the approximate solution is indeed accurate. Various results and comparisons are presented and discussed. Lastly, the analysis was also extended to solve the problem of thin flow over a cylinder and the fundamental differences between the flow over a sphere and that over a cylinder have been identified and explained. The technique and the approach adopted can be used to model and understand similar thin flows that occur in other settings.

Keywords: analytical, incompressible, thin flow, viscous.
\end{abstract}

\section{INTRODUCTION}

The globe fountain consists of a sphere with a hole at the top whereby water is pumped out at a constant rate. These can be found at garden centers and are sold for decorative purposes. A typical globe fountain is illustrated below in Fig. 1. The diagram shows a pillbox formed at the top of the sphere as a result of the water flowing out of the hole. Although the fluid mechanics associated with the formation of the pillbox is very interesting, our focus in this study is in the subsequent thin layer flow following the pillbox; we will model the flow as it spreads over the sphere.

Another interesting fountain, known as the "kugel fountain", involves a massive granite sphere which floats and spins on a thin film of flowing water which is pumped out of a hole at the base of the fountain. The dynamics of this fountain have recently been analyzed by Snoeijer \& van der Weele [2] using lubrication theory.

Related to the globe fountain is the study conducted by Takagi \& Huppert [3] whereby a constant volume of fluid was released at the top of a sphere. Lubrication theory was used to show that the fluid thickness remains constant along the surface of the sphere but decreases with time $(t)$ according to $\sqrt{ } t$. Analytical solutions were found to agree well with their experiments. They also investigated the onset of instability of the advancing front as it split into a series of rivulets.

Since the globe fountain flow involves a thin fluid layer, this property will be exploited in our analysis. Because thin fluid layers occur in a variety of settings $[4,5]$, they have received 


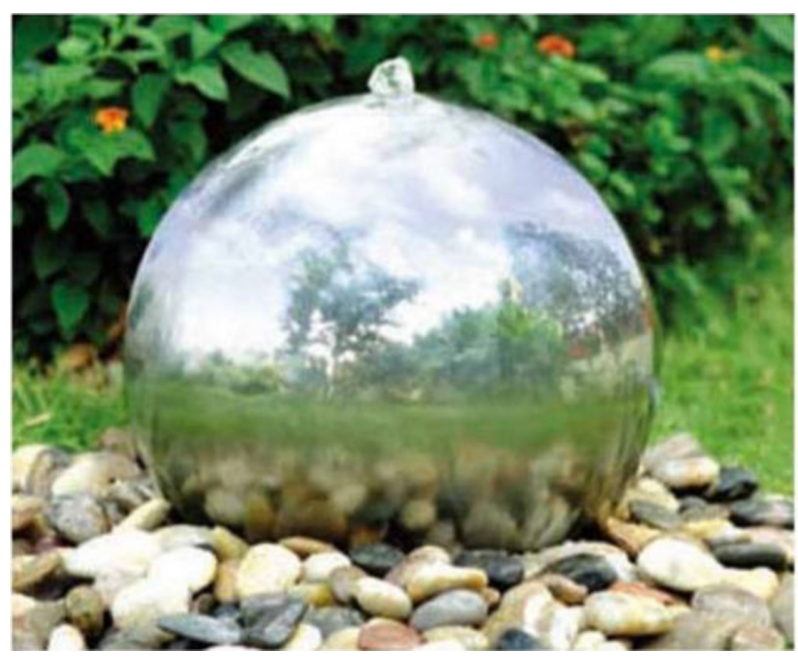

Figure 1: A typical globe fountain [1].

considerable attention over the years. In our investigation, we derive an approximate solution for the steady-state flow based on the assumed smallness of a parameter related to the characteristic thickness of the fluid layer. The study is organized as follows. In section 2 we present the governing equations and the corresponding boundary conditions. When cast in dimensionless form a small parameter, defined as the ratio of the fluid layer thickness to the radius of the sphere, naturally appears. In Section 3, we then construct an approximate analytical solution for the steady-state flow based on the small parameter. The analytical results are then discussed in Section 4 and a summary is given in Section 5.

\section{GOVERNING EQUATIONS}

We consider the laminar steady flow of a viscous incompressible isothermal Newtonian fluid exiting a small hole at the top of a sphere and spilling over the surface. Formulated in spherical coordinates $(r, \theta, \phi)$ with the hole oriented about the polar axis $\theta=0$ and assuming azimuthal symmetry, the governing steady-state Navier-Stokes equations become

$$
\begin{gathered}
\frac{\partial}{\partial r}\left(r^{2} v \sin \theta\right)+\frac{\partial}{\partial \theta}(r u \sin \theta)=0 \\
v \frac{\partial v}{\partial r}+\frac{u}{r} \frac{\partial v}{\partial \theta}-\frac{u^{2}}{r}=-\frac{1}{\rho} \frac{\partial P}{\partial r}-g \cos \theta+\frac{\mu}{\rho r^{2}} \times \\
{\left[\frac{\partial}{\partial r}\left(r^{2} \frac{\partial v}{\partial r}\right)+\frac{1}{\sin \theta} \frac{\partial}{\partial \theta}\left(\sin \theta \frac{\partial v}{\partial \theta}\right)-\frac{2}{\sin \theta} \frac{\partial}{\partial \theta}(u \sin \theta)-2 v\right],} \\
+\frac{\mu}{\rho r^{2}}\left[\frac{\partial}{\partial r}\left(r^{2} \frac{\partial u}{\partial r}\right)+\frac{u}{r} \frac{\partial u}{\sin \theta} \frac{\partial}{\partial \theta}\left(\sin \theta \frac{\partial u}{\partial \theta}\right)+2 \frac{\partial u}{\partial \theta}-\frac{u}{\sin ^{2} \theta}\right]
\end{gathered}
$$


Here, the flow variables $u, v$ denote the velocity components in the $\theta$ and radial directions, respectively, while $P$ refers to the pressure. The fluid property $\mu / \rho$ represents the kinematic viscosity while $\mu$ is the dynamic viscosity and $\rho$ is the fluid density whereas $g$ is the acceleration due to gravity. To cast the equations in dimensionless form, we scale the thickness of the fluid layer by the Nusselt thickness, $H$, which for a vertical incline (i.e. $\theta=\pi / 2$ where $\theta$ is the angle of inclination) is given by

$$
H^{3}=\frac{3 \mu Q}{\rho g},
$$

where $Q$ is the constant flow rate exiting the small hole. For a fluid layer having a width of unity $Q=U H$ where $U$ is the velocity scale.

We next introduce the coordinate $y$ which is related to $r$ through the relation $r=R+y$ with $R$ referring to the radius of the sphere. This can be scaled as

$$
\frac{r}{R}=1+\delta\left(\frac{y}{H}\right)
$$

where the dimensionless parameter $\delta=H / R$ « 1 denotes the shallowness parameter. With this scaling the dimensionless flow variables and coordinate $y$ are given by

$$
(u, v, P, y) \rightarrow\left(U \tilde{u}, \delta U \widetilde{v}, \rho U^{2} \widetilde{P}, H \tilde{y}\right),
$$

where the tilde denotes a dimensionless quantity. In dimensionless form eqns (1)-(3) become

$$
\begin{gathered}
\frac{\partial}{\partial y}\left[(1+\delta)^{2} v \sin \theta\right]+\frac{\partial}{\partial \theta}[(1+\delta y) u \sin \theta]=0, \\
\delta^{2} v \frac{\partial v}{\partial y}+\frac{\delta^{2} u}{(1+\delta y)} \frac{\partial v}{\partial \theta}-\frac{\delta u^{2}}{(1+\delta y)}=-\frac{\partial P}{\partial y}-\frac{3 \cos \theta}{R e}+\frac{\delta}{\operatorname{Re}(1+\delta y)^{2}} \times \\
{\left[\frac{\partial}{\partial y}\left((1+\delta y)^{2} \frac{\partial u}{\partial y}\right)+\frac{\delta^{2}}{\sin \theta} \frac{\partial}{\partial \theta}\left(\sin \theta \frac{\partial}{\partial \theta}\right)-\frac{2 \delta}{\sin \theta}(u \sin \theta)-2 \delta^{2} v\right],} \\
\delta v \frac{\partial u}{\partial y}+\frac{\delta u}{(1+\delta y)} \frac{\partial u}{\partial \theta}+\frac{\delta^{2} u v}{(1+\delta y)}=-\frac{\delta}{(1+\delta y)} \frac{\partial P}{\partial \theta}+\frac{3 \sin \theta}{\operatorname{Re}}+\frac{1}{\operatorname{Re}(1+\delta y)^{2}} \times \\
{\left[\frac{\partial}{\partial y}\left((1+\delta y)^{2} \frac{\partial u}{\partial y}\right)+\frac{\delta^{2}}{\sin \theta} \frac{\partial}{\partial \theta}\left(\sin \theta \frac{\partial u}{\partial \theta}\right)+2 \delta^{3} \frac{\partial v}{\partial \theta}-\frac{\delta^{2} u}{\sin ^{2} \theta}\right],}
\end{gathered}
$$

where $R e=Q \rho / \mu$ denotes the Reynolds number, and the tildes have been suppressed for notational convenience.

Along the air-fluid interface, we apply dynamic conditions which ensure the continuity of normal and tangential stress. In dimensionless form, these conditions along the steady free surface $y=\eta(\theta)$ become 


$$
\begin{gathered}
P-P_{a}=\delta W e\left[\frac{3 \delta^{2}\left(\eta^{\prime}\right)^{2}+2(1+\delta \eta)^{2}-\delta(1+\delta \eta) \eta^{\prime \prime}}{\left[(1+\delta \eta)^{2}+\delta^{2}\left(\eta^{\prime}\right)^{2}\right]^{3 / 2}}\right. \\
\left.-\frac{\delta \eta^{\prime} \cot \theta}{(1+\delta \eta) \sqrt{(1+\delta \eta)^{2}+\delta^{2}\left(\eta^{\prime}\right)^{2}}}\right]+\frac{2 \delta}{\operatorname{Re}\left[(1+\delta \eta)^{2}+\delta^{2}\left(\eta^{\prime}\right)^{2}\right]}\left[(1+\delta \eta)^{2} \frac{\partial u}{\partial y}\right. \\
\left.+\frac{\delta^{2}\left(\eta^{\prime}\right)^{2}}{(1+\delta \eta)}\left(\delta u+\frac{\partial u}{\partial \theta}\right)-(1+\delta \eta) \eta^{\prime}\left(\frac{\partial u}{\partial y}+\frac{\delta}{(1+\delta \eta)}\left(\delta \frac{\partial v}{\partial \theta}-u\right)\right)\right] \\
{\left[(1+\delta \eta)^{2}-\delta^{2}\left(\eta^{\prime}\right)^{2}\right]\left(\frac{\partial u}{\partial y}+\frac{\delta}{(1+\delta \eta)}\left(\delta \frac{\partial v}{\partial \theta}-u\right)\right)} \\
+2 \delta(1+\delta \eta) \eta^{\prime}\left(\frac{\partial u}{\partial y}+\frac{\delta}{(1+\delta \eta)}\left(\delta \frac{\partial v}{\partial \theta}-u\right)\right)=0
\end{gathered}
$$

where $W e=\sigma H /\left(\rho \mathrm{Q}^{2}\right)$ is the Weber number with $\sigma$ denoting surface tension, $P_{a}$ the constant ambient air pressure, $\eta=h / H$ with $h$ being the thickness of the fluid layer, and the prime denotes differentiation with respect to $\theta$. In addition, we apply the kinematic condition along the free surface $y=\eta(\theta)$ given by

$$
v=\frac{u}{(1+\delta \eta)} \eta^{\prime}
$$

We also apply the following no-slip and impermeability conditions on the surface of the sphere

$$
u=v=0 \text { at } y=0
$$

\section{APPROXIMATE ANALYTICAL SOLUTION}

For small $\delta$, an approximate analytical solution can be constructed by expanding the flow variables in the following series

$$
\begin{aligned}
u(y, \theta) & =u_{0}(y, \theta)+\delta u_{l}(y, \theta)+\cdots, \\
v(y, \theta) & =v_{0}(y, \theta)+\delta v_{l}(y, \theta)+\cdots, \\
P(y, \theta) & =P_{0}(y, \theta)+\delta P_{1}(y, \theta)+\cdots, \\
\eta(\theta) & =\eta_{0}(\theta)+\delta \eta_{l}(\theta)+\cdots .
\end{aligned}
$$

Substituting these expansions into the equations of motion and expanding the dynamic conditions in powers of $\delta$ leads to a hierarchy of problems.

The leading-order problem is governed by the system

$$
\begin{aligned}
& \frac{\partial P_{0}}{\partial y}=-\frac{3 \cos \theta}{R e}, \\
& \frac{\partial^{2} u_{0}}{\partial y^{2}}=-3 \sin \theta,
\end{aligned}
$$




$$
\sin \theta\left(\frac{\partial v_{0}}{\partial y}+\frac{\partial u_{0}}{\partial \theta}\right)+u_{0} \cos \theta=0
$$

subject to

$$
\begin{aligned}
& P_{0}=P_{a}, \frac{\partial u_{0}}{\partial y}=0 \text { at } y=\eta_{0}, \\
& u_{0}=v_{0}=0 \text { at } y=0 .
\end{aligned}
$$

The solutions are easily obtained and are given by

$$
\begin{gathered}
P_{0}(y, \theta)=P_{a}+\frac{3 \cos \theta}{\operatorname{Re}}\left(\eta_{0}-y\right), \\
u_{0}(y, \theta)=\frac{3}{2} y \sin \theta\left(2 \eta_{0}-y\right), \\
v_{0}(y, \theta)=-\frac{y^{2}}{2}\left(6 \eta_{0} \cos \theta+3 \eta_{0}^{\prime} \sin \theta-2 y \cos \theta\right) .
\end{gathered}
$$

Thus to leading order, the pressure is hydrostatic and the velocity in the $\theta$ direction, $u_{0}$, has a parabolic profile in $y$ which is consistent with flow down an inclined surface at an angle of $\theta$ with the horizontal. Further, the velocity component $u_{0}$ is symmetric about the plane $\theta=\pi / 2$. The leading order term for the unknown free surface can be determined by applying the kinematic condition which when transferred from $y=\eta$ to $y=\eta_{0}$ takes the form

$$
v_{0}=u_{0} \eta_{0}^{\prime} \text { at } y=\eta_{0} .
$$

This leads to the differential equation

$$
\eta_{0}^{\prime}=-\frac{2 \eta_{0} \cos \theta}{3 \sin \theta}
$$

which can be solved to yield

$$
\eta_{0}(\theta)=\frac{C}{\sin ^{2 / 3} \theta}
$$

In order to determine the arbitrary constant $C$ a boundary condition must be provided. We note that the solution for $\eta_{0}$ is singular at $\theta=0, \pi$.

As fluid flows out of the hole at the top of the sphere, a pillbox will be formed. Since our focus is in the subsequent flow following the pillbox, we propose the generic boundary condition

$$
\eta_{0}=h_{0} \text { at } \theta=\theta_{0},
$$

and will treat $h_{0}$ and $\theta_{0}$ as free dimensionless parameters. Thus, $C=h_{0} \sin ^{2 / 3} \theta_{0}$ and the $\sin$ gularity at $\theta=0$ is removed. Since we expect the flow to separate from the surface before reaching the bottom of the sphere, the singularity at $\theta=\pi$ is also resolved. 
The $O(\delta)$ problem satisfies the system

$$
\begin{gathered}
\frac{\partial^{2} u_{1}}{\partial y^{2}}=\operatorname{Re} \frac{\partial}{\partial \theta}\left(P_{0}+\frac{u_{0}^{2}}{2}\right)+\left(\operatorname{Rev}_{0}-2\right) \frac{\partial u_{0}}{\partial y}, \\
\sin \theta\left(\frac{\partial v_{1}}{\partial y}+\frac{\partial u_{1}}{\partial \theta}\right)+u_{1} \cos \theta= \\
-\sin \theta\left(2 y \frac{\partial v_{0}}{\partial y}+y \frac{\partial u_{0}}{\partial \theta}+2 v_{0}\right)-y u_{0} \cos \theta
\end{gathered}
$$

subject to

$$
\begin{gathered}
\frac{\partial u_{1}}{\partial y}+\eta_{1} \frac{\partial^{2} u_{0}}{\partial y^{2}}=u_{0}-2 \eta_{0} \frac{\partial u_{0}}{\partial y}-2 \eta_{0}^{\prime} \frac{\partial v_{0}}{\partial y} \text { at } y=\eta_{0}, \\
u_{1}=v_{1}=0 \text { at } y=0 .
\end{gathered}
$$

Lastly, the kinematic condition applied at $y=\eta_{0}$ furnishes the following differential equation for the free surface correction, $\eta_{1}$

$$
u_{0} \eta_{1}^{\prime}+\left(\eta_{0}^{\prime} \frac{\partial u_{0}}{\partial y}-\frac{\partial v_{0}}{\partial y}\right) \eta_{1}=v_{1}+\eta_{0} \eta_{0}^{\prime} u_{0}-\eta_{0}^{\prime} u_{1}
$$

subject to

$$
\eta_{1}=0 \text { at } \theta=\theta_{0} .
$$

The solution to the $O(\delta)$ problem is significantly more complicated and was obtained using the Maple Computer Algebra system. The solution for $u_{1}$ is

given by

$$
\begin{gathered}
u_{1}(y, \theta)=\frac{y}{40}\left(-\operatorname{Re} y^{5} \sin \theta \cos \theta+6 C \operatorname{Rey}^{4} \sin ^{1 / 3} \theta \cos \theta+15 C \operatorname{Re}_{0}^{\prime} y^{3} \sin ^{4 / 3} \theta\right. \\
\left.+60 y^{2} \sin \theta+60 y\left[\eta_{0}^{\prime} \cos \theta-3 C \sin ^{1 / 3} \theta\right]+F(\theta)\right)
\end{gathered}
$$

where

$$
\begin{aligned}
& F(\theta)=120 \eta_{1} \sin \theta+240 C^{2} \sin ^{-4 / 3} \theta\left[\eta_{0}^{\prime} \cos \theta+\sin \theta\right]-60 C^{4} R e \eta_{0}^{\prime} \sin ^{-2 / 3} \theta \\
& +240 C\left(\eta_{0}^{\prime}\right)^{2} \sin ^{1 / 3} \theta-120 C \eta_{0}^{\prime} \sin ^{-2 / 3} \theta \cos \theta-24 C^{5} R e \sin ^{-7 / 3} \theta \cos \theta
\end{aligned}
$$

The solution for $\eta_{1}$ can be expressed in the following form

$$
\eta_{1}(\theta)=-\frac{512 C^{2}}{945 \sin ^{2 / 3} \theta} \int_{\theta_{0}}^{\theta} f(\alpha) d \alpha
$$

and can be integrated numerically. Here, $f(\alpha)$ is given by

$$
\begin{gathered}
f(\alpha)=\sin ^{23 / 3} a\left(-180 C^{3} R e \sin ^{2 / 3} a \cos 2 a-396 C^{3} \operatorname{Resin}^{2 / 3} a+280 C \cos 3 a\right. \\
\left.+2520 C \cos a+385 \sin ^{2 / 3} a \cos 3 a-2625 \sin ^{2 / 3} a \cos \alpha\right) / \\
(462+\cos 12 a-12 \cos 10 a+66 \cos 8 \alpha-220 \cos 6 a+495 \cos 4 a-792 \cos 2 \alpha) .
\end{gathered}
$$




\section{RESULTS AND DISCUSSION}

Figure 2 shows the leading-order and first-order correction to the free surface using $R e=1$, $\delta=0.1, \theta_{0}=0.2$ and $h_{0}=0.5$ while Fig. 3 illustrates the free surface for $\theta_{0}<\theta<\pi / 2$ in Cartesian coordinates. We have intentionally chosen a thicker fluid layer in these plots to demonstrate that the impact of the correction term is relatively small. For thinner fluid layers, the difference between the leading-order and first-order correction is much smaller and hardly noticeable. The fluid thickness decreases as $\theta$ increases from $\theta_{0}$ and attains a minimum near the equator, $\theta=\pi / 2$, and then increases again almost symmetrically. The reason for this is simple. As the fluid flows over the sphere toward the equator, it is spreading over a larger surface area. In fact, the rate at which the surface area is increasing is such that the average streamwise speed decreases. This can be seen by computing the dimensionless flow rate per unit width, $\hat{Q}$, and dimensionless average streamwise velocity, $\hat{U}$, using the leading-order solution as follows

$$
\hat{Q}=\int_{0}^{\eta_{0}} u_{0}(y, \theta) d y=\eta_{0}^{3} \sin \theta, \hat{U}=\frac{\hat{Q}}{\eta_{0}}=\eta_{0}^{3} \sin \theta
$$

Substituting $\eta_{0}=C / \sin ^{2 / 3} \theta$ yields $\hat{Q}=C^{3} / \sin \theta$ and $\hat{U}=C^{2} / \sin ^{1 / 3} \theta$ which shows that both the flow rate and average speed decrease as $\theta$ increases from $\theta_{0}$ to $\pi / 2$, and hence, a decrease in fluid thickness. We note that $\hat{Q} \sin \theta$ is constant as it should be since this represents the total flow rate over the entire sphere at a given angle $\theta$.

Plotted in Fig. 4 is the leading-order, $u_{0}$, and first-order correction, $u_{1}$, to the streamwise velocity profile using $R e=1, \delta=0.1, \theta_{0}=0.2$ and $h_{0}=1$ at $\theta=\pi / 4$ while Fig. 5 compares the velocity profile $u=u_{0}+\delta u_{1}$ at $\theta=\pi / 4$ with that at $\theta=\pi / 2$. Again, we have intentionally

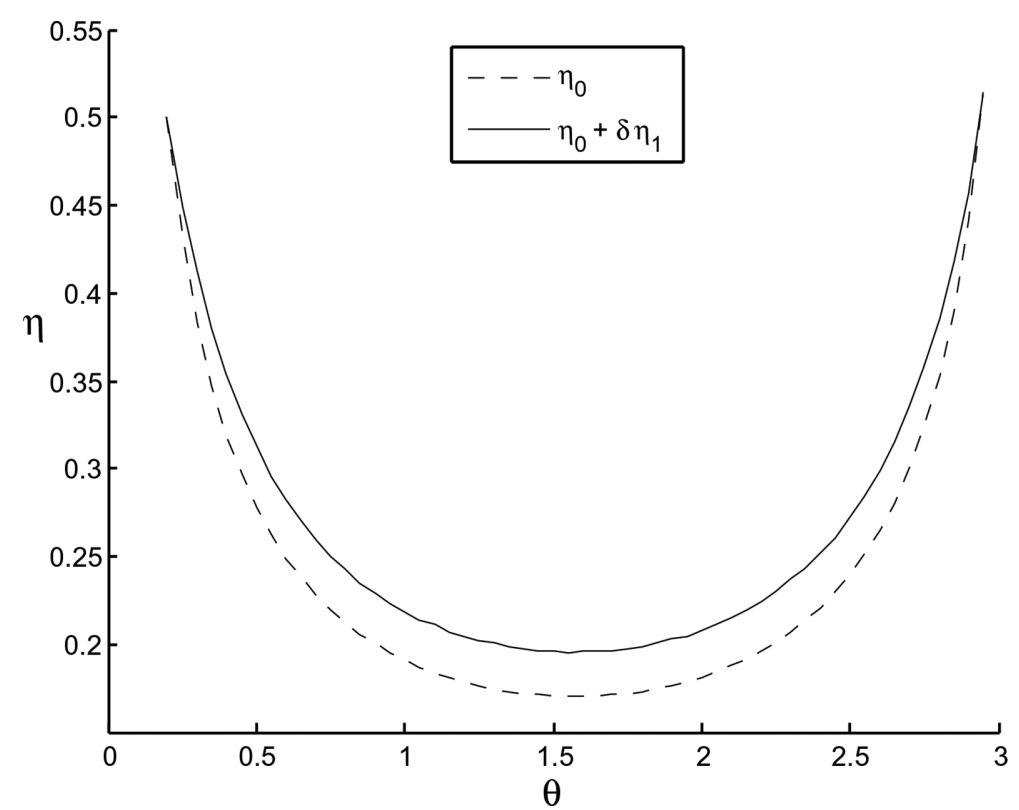

Figure 2: The leading-order, $\eta_{0}$, and first-order correction, $\eta_{1}$, to the free surface with $\operatorname{Re}=1, \delta=0.1, \theta_{0}=0.2$ and $h_{0}=0.5$. 


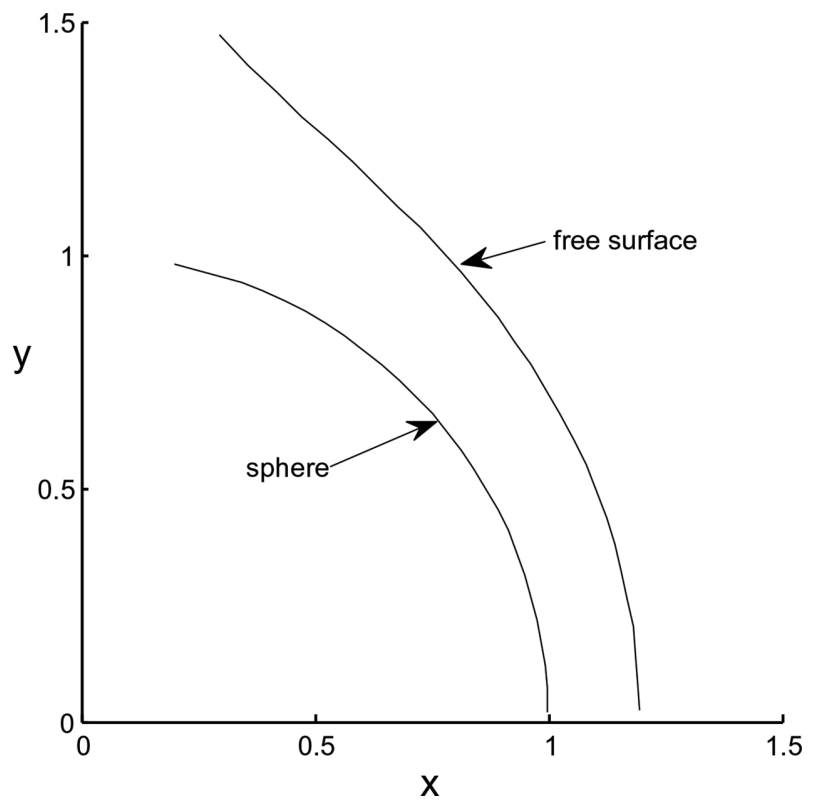

Figure 3: The free surface $\eta=\eta_{0}+\delta \eta_{1}$ shown in Cartesian coordinates with $R e=1, \delta=0.1$, $\theta_{0}=0.2$ and $h_{0}=0.5$.

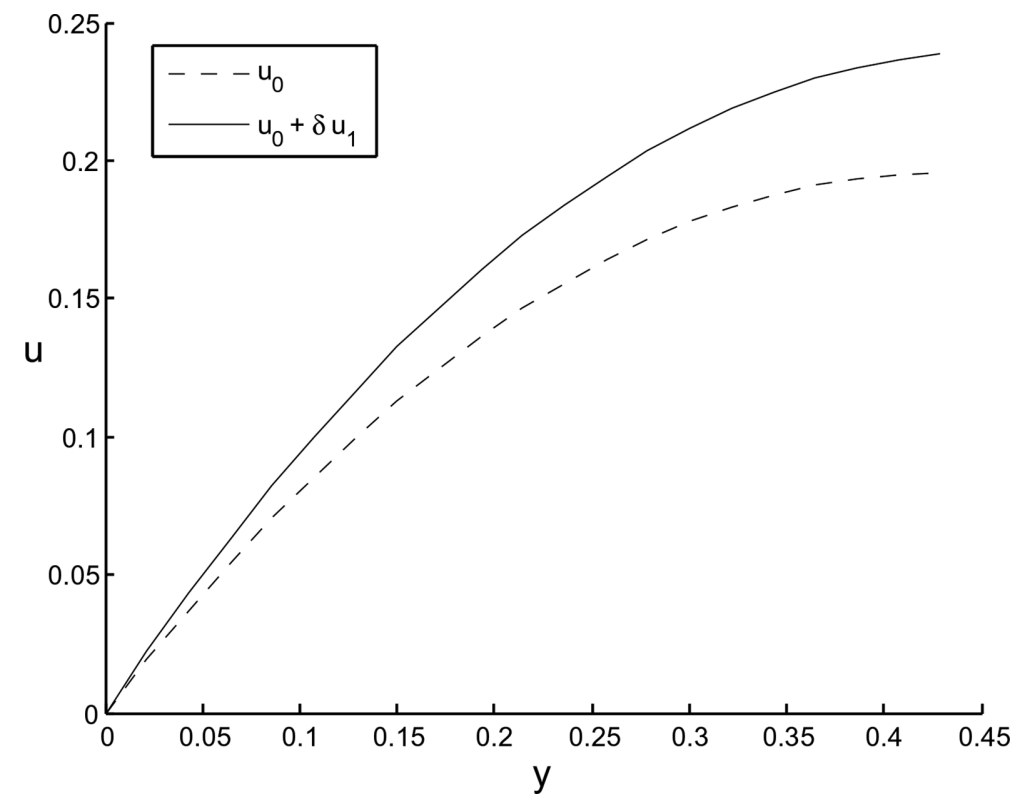

Figure 4: The leading-order, $u_{0}$, and first-order correction, $u_{1}$, to the velocity profile with $\operatorname{Re}=1, \delta=0.1, \theta_{0}=0.2$ and $h_{0}=1$ at $\theta=\pi / 4$. 
chosen a thick fluid layer to illustrate the relative size of the correction term. In general, the velocity profiles are very similar. The only differences are in the maximum velocity and thickness of the fluid layer. As previously noted, the fluid thickness decreases as it flows over the sphere, and Fig. 5 demonstrates that the maximum velocity, which occurs at the free surface, also decreases, and hence, the average speed decreases.

One method of estimating where the fluid separates from the surface is to use the zerostress condition at the surface, that is, set

$$
\frac{\partial u}{\partial y}=0 \text { at } y=0 .
$$

Using $u=u_{0}$ leads to $3 \eta_{0}(\theta) \sin \theta=0$ and solving this yields the separation angle $\theta_{s}=\pi$ which supports the claim made earlier. Including the first-order correction $u=u_{0}+\delta u_{1}$ requires solving the equation

$$
3 \eta_{0}(\theta) \sin \theta+\frac{\delta}{40} F(\theta)=0,
$$

to determine the separation angle $\theta_{s}$ where $F(\theta)$ was previously defined. Our calculations revealed that $\theta_{s}$ occurs near the bottom of the sphere and the dependence on $R e$ and $\delta$ is negligible. There was some dependence on $h_{0}$ and $\theta_{0}$. For example, with $h_{0}=1$ and $\theta_{0}=0.2$ we obtained $\theta_{s} \approx 3.045$ whereas with $h_{0}=0.5$ and $\theta_{0}=0.3$ we obtained $\theta_{s} \approx 3.085$. The observed trend was that for a fixed value of $h_{0}\left(\theta_{0}\right)$ the value of $\theta_{s}$ approached $\pi$ as $\theta_{0}\left(h_{0}\right)$ decreased.

When the calculation was repeated for the problem of thin flow over a cylinder, very similar stream-wise velocity profiles were obtained. However, the free surface variation was

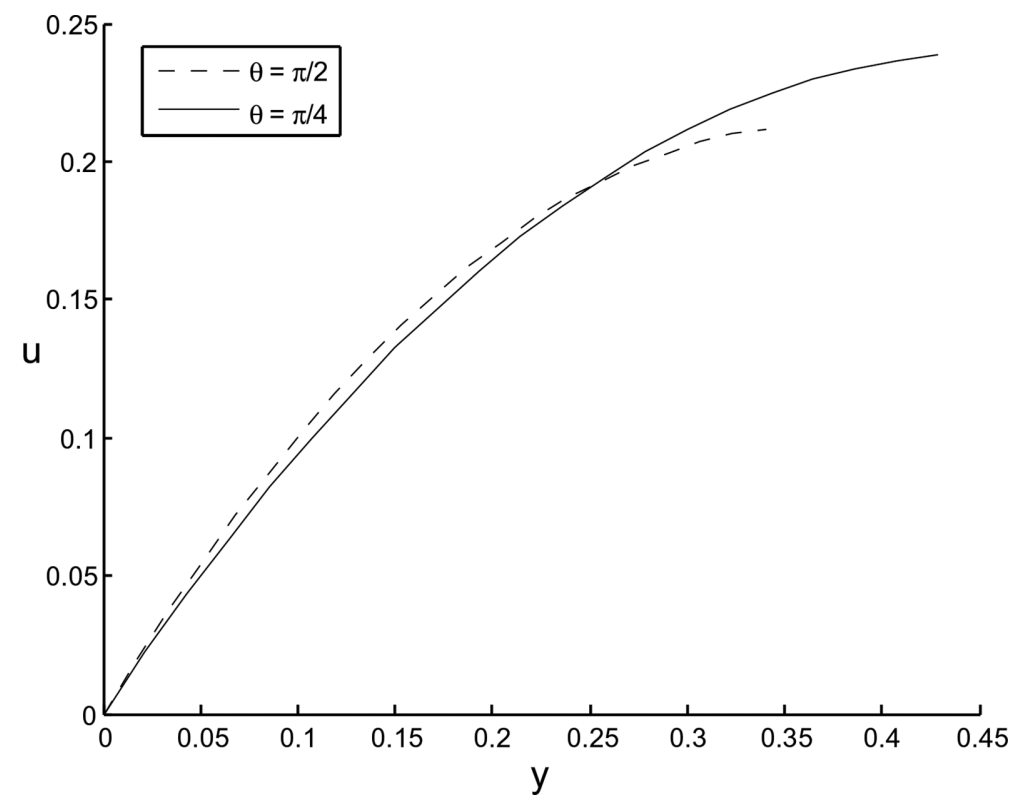

Figure 5: The velocity profile $u=u_{0}+\delta u_{1}$ using $R e=1, \delta=0.1, \theta_{0}=0.2$ and $h_{0}=1$ at $\theta=\pi / 4$ and $\theta=\pi / 2$. 
noticeably different. Contrasted in Fig. 6 are the free surface plots for the cylinder and sphere using $\operatorname{Re}=1, \delta=0.1, \theta_{0}=0.2$ and $h_{0}=0.5$. We see that the free surface varies more rapidly with $\theta$ for the sphere than it does for the cylinder. Since the surface area remains constant as the fluid spreads over the cylinder, the decrease in fluid thickness must be a result of an increasing average speed. Indeed, for flow over a cylinder, we find that $\hat{Q}=D^{3}$ and $\hat{U}=\hat{Q} / \eta_{0}$ $=D^{2} \sin ^{1 / 3} \theta$ where $D=h_{0} \sin ^{1 / 3} \theta_{0}$. This confirms that the flow rate remains constant and the average speed increases. Expressions for $\eta_{0}(\theta)$ and $\eta_{1}(\theta)$ for the cylinder are given by

$$
\begin{gathered}
\eta_{0}(\theta)=\frac{D}{\sin ^{1 / 3} \theta}, \\
\eta_{1}(\theta)=-\frac{D^{2}}{7560 \sin ^{1 / 3} \theta} \int_{\theta_{0}}^{\theta} \frac{1}{\sin ^{11 / 3} a}\left(245 \sin ^{1 / 3} a \cos 3 \alpha-2205 \sin ^{1 / 3} a \cos \alpha\right. \\
\left.+70 D \cos 3 a+1050 D \cos \alpha+72 D^{3} R e \sin ^{4 / 3} a \cos 2 \alpha+504 D^{3} R e \sin ^{4 / 3} \alpha\right) d \alpha .
\end{gathered}
$$

\section{CONCLUSIONS}

Discussed in this work was an analytical investigation of the steady flow of a thin fluid layer over a sphere resulting from a constant discharge from a small hole at the top of the sphere. An approximate analytical solution was derived which for thin flows is judged to be accurate. The fluid thickness decreases as it flows over the sphere due to the increasing surface area and then increases almost symmetrically for $\theta>\pi / 2$. The leading-order stream-wise velocity profile is parabolic and bears a close resemblance to that of the well-known problem

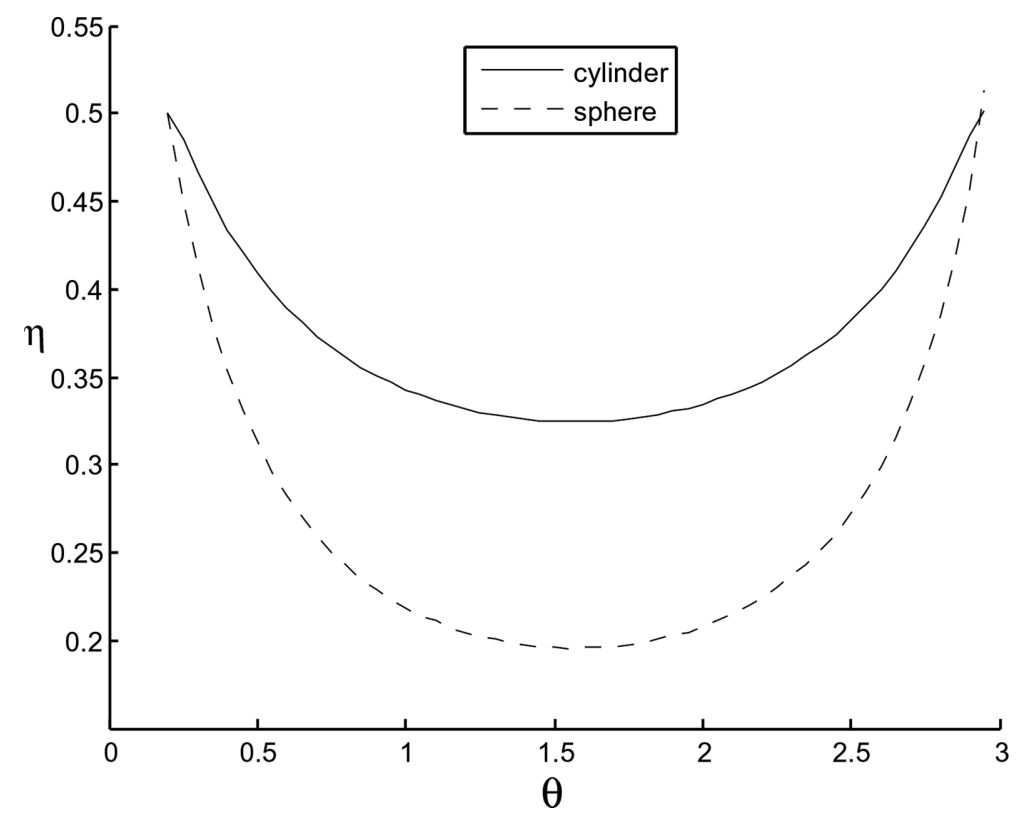

Figure 6: The free surface $\eta=\eta_{0}+\delta \eta_{1}$ for the cylinder and sphere with $R e=1, \delta=0.1$, $\theta_{0}=0.2$ and $h_{0}=0.5$. 
of flow down an incline. The calculation was repeated for the case of thin flow over a cylinder, and the velocity profiles obtained were similar in the form to those for the sphere. However, noticeable differences in the free surface occurred where it was discovered that the change in fluid layer thickness was much greater for the sphere than it was for the cylinder. This is because the average stream-wise velocity increases as the fluid flows over the cylinder and since the flow rate is independent of the angle $\theta$ the thickness must decrease accordingly. For the sphere, on the other hand, both the flow rate and the average streamwise velocity decrease as the fluid flows over the surface which leads to a more rapid decrease in fluid layer thickness. Although the derived solutions break down as $\theta \rightarrow \pi$, according to our predictions the flow separates from the surface before reaching the bottom, and hence, the solutions are not valid near $\theta=\pi$.

\section{ACKNOWLEDGMENTS}

Financial support for this research was provided by the Natural Sciences and Engineering Research Council of Canada and the Faculty of Mathematics at the University of Waterloo.

\section{REFERENCES}

[1] http://www.ebay.com/sch/i.html?_nkw=sphere+water+fountain

[2] Snoeijer, J.H. \& van der Weele, K., Physics of the granite sphere fountain. American Journal of Physics, 82, pp. 1029-1039, 2014. http://dx.doi.org/10.1119/1.4886365

[3] Takagi, D. \& Huppert, H.E., Flow and instability of thin films on a cylinder and sphere. Journal of Fluid Mechanics, 647, pp. 221-238, 2010. http://dx.doi.org/10.1017/S0022112009993818

[4] Kalliadasis, S., Ruyer-Quil, C., Scheid, B. \& Velarde, M.G., Falling Liquid Films, Springer-Verlag: London, UK, 2012. http://dx.doi.org/10.1007/978-1-84882-367-9

[5] Craster, R.V. \& Matar, O.K., Dynamics and stability of thin liquid films. Reviews of Modern Physics, 81, pp. 1131-1198, 2009. http://dx.doi.org/10.1103/RevModPhys.81.1131 